\title{
Synthesis of All Low-Energy Stereoisomers of the Tris(pyrrolidinoindoline) Alkaloid Hodgkinsine and Preliminary Assessment of Their Antinociceptive Activity
}

\author{
Jeremy J. Kodanko ${ }^{\dagger}$, Sheldon Hiebert ${ }^{\dagger}$, Emily A. Peterson ${ }^{\dagger}$, Leonard Sung ${ }^{\dagger}$, Larry E. Overman ${ }^{* \dagger}$, \\ Viviane de Moura Linck ${ }^{*}$, Greice Catrine Goerck ${ }^{\star}$, Tânia Alves Amador ${ }^{\dagger}$, Mirna Bainy Leal ${ }^{*}$ and Elaine \\ Elisabetsky *t
}

\section{Supporting Information \\ (29 pages)}

Part A. General experimental details and experimental details for the conversion of 4 to 6: S1-2.

Part B. Tables of the effects of hodgkinsine and its stereoisomers in the tail-flick and capsaicin pain models and reversibility in the tail flick assay by Naloxone: S3-4.

Part C. ${ }^{1} \mathrm{H}$ and ${ }^{13} \mathrm{C}$ NMR spectra for all new compounds; all compounds are complex mixtures of conformational stereoisomers: S5-24.

Part D. CD spectra $\left(\mathrm{CHCl}_{3}\right)$ of hodgkinsine and its stereoisomers: S25-29.

\section{Part A.}

General Procedures for Synthesis: Reactions were carried out using flame-dried glassware under a nitrogen atmosphere unless otherwise stated. Tetrahydrofuran (THF), diethyl ether (Et $2 \mathrm{O}$ ), dichloromethane $\left(\mathrm{CH}_{2} \mathrm{Cl}_{2}\right)$, and acetonitrile $\left(\mathrm{CH}_{3} \mathrm{CN}\right)$ were degassed with argon and then passed through columns of anhydrous alumina to remove $\mathrm{H}_{2} \mathrm{O}$. N,N,N',N'-Tetramethylethylenediamine (TMEDA), $N$-methyl-2-pyrrolidinone (NMP), and 1,2,2,6,6-pentamethylpiperidine (PMP) were distilled from $\mathrm{CaH}_{2}$ prior to use. Concentration of $s$-BuLi was determined by titration against $N$ benzylbenzamide according to Burchat, A. F.; Chong, M. J.; Nielsen, N. J. Organomet. Chem. 1997, 542, 281-283. Compound $\mathbf{7}^{1}$ and $\mathbf{1 2}^{2}$ were prepared as previously reported. All other commercial reagents were used as received unless otherwise indicated.

\footnotetext{
${ }^{\dagger}$ Department of Chemistry, 1102 Natural Sciences II, University of California, Irvine, California 92697-2025

* Laboratório de Etnofarmacologia, ICBS, Universidade Federal do Rio Grande do Sul, Rua Sarmento Leite 500/202, 90050170, Porto Alegre, Brazil.

${ }^{\dagger}$ Faculdade de Farmácia, Universidade Federal do Rio Grande do Sul, Av. Ipiranga 2752, 90610-000, Porto Alegre, Brazil.

${ }^{1}$ Overman, L. E.; Larrow, J. F.; Stearns, B. A.; Vance, J. M. Angew. Chem., Int. Ed. 2000, 39, $213-215$.

${ }^{2}$ Lebsack, A. D.; Link, J. T.; Overman, L. E.; Stearns, B. A. J. Am. Chem. Soc. 2002, 124, 9008-9009.
} 
General Procedures for Measuring Antinociceptive Activity. Experiments were performed using male (CF1) adult albino mice, 2.5 months of age (30-35 g) received from Fundação Estadual de Produção e Pesquisa em Saúde (FEPPS). Animals were maintained in the animal facility at UFRGS under controlled environmental conditions $\left(22 \pm 1{ }^{\circ} \mathrm{C}, 12 \mathrm{hr}\right.$-light/dark cycle, free access to food and water), for at least two weeks before the experiments. All procedures were carried out in accordance with institutional policies on experimental animals handling, which follow the NIH guidelines $(N I H$ Guide for Care and Use of Laboratory Animals, NIH publication no. 85-23, 1985). Natural hodgkinsine was isolated from $P$. colorata flowers as detailed elsewhere; ${ }^{3}$ MK-801 was acquired from RBI. Capsaicin and naloxone were acquired from Sigma-Aldrich and morphine from commercial sources $\left(\right.$ Dimorph $^{\circledR}$, Cristália Indústria Farmacêutica LTDA., Brazil). Hodgkinsine and derivatives were diluted in DMSO (10\%), except in experiments where hodgkinsine was transformed into salt by adding stoichiometric amounts of $\mathrm{HCl}$ and diluted in distilled water. Analgesia was assessed using a tail-flick apparatus (Albrasch Electronic Equipment) by the general method of Netto and co-workers, ${ }^{4}$ as previously described. ${ }^{5}$ Capsaicin-induced pain was determined according to the procedure of Corrêa and co-workers. ${ }^{6}$

Conversion of 4 to idiospermuline (6). MeI (100 mL, $0.0096 \mathrm{mmol}, 0.096 \mathrm{M}$ in THF) was added to a solution of $4(2.0 \mathrm{mg}, 0.0039 \mathrm{mmol})$ and THF $(0.5 \mathrm{~mL})$ at $-78{ }^{\circ} \mathrm{C}$. NaHMDS $(100 \mathrm{~mL}, 0.012 \mathrm{mmol}$, $0.12 \mathrm{M}$ in THF) was then added, and the reaction was stirred for $5 \mathrm{~min}$ at $-78{ }^{\circ} \mathrm{C}$, at which point the cold bath was removed and the reaction was allowed to warm to room temperature. After $30 \mathrm{~min}$, the reaction was quenched by addition of $\mathrm{NaHCO}_{3}(1 \mathrm{~mL}$, saturated aqueous). The aqueous layer was extracted with EtOAc $(2 \mathrm{~mL})$ and $\mathrm{CHCl}_{3}(2 \times 2 \mathrm{~mL})$, and the combined organic layers were dried $\left(\mathrm{Na}_{2} \mathrm{SO}_{4}\right)$ and evaporated. TLC and ESMS analysis showed a mixture of starting material, monomethylated and dimethylated products. The crude mixture was resubjected to the reaction conditions using MeI (100 mL, $0.0096 \mathrm{mmol}, 0.096 \mathrm{M}$ in THF), NaHMDS (25 mL, 0.025 mmol, $1.0 \mathrm{M}$ in THF), and THF $(0.5 \mathrm{~mL})$. The crude product was purified by preparative TLC (7:3:1 benzene/EtOAc/Et ${ }_{2} \mathrm{NH}, 0.25 \mathrm{~mm}$ silica plate from E. Merck) to give 6 as a colorless solid (2.0 mg, 95\%). Spectral ( ${ }^{1} \mathrm{H} \mathrm{NMR}$ in $\mathrm{CDCl}_{3}$ ), TLC and HPLC (Zorbax Extend 5 mm C-18 column, 250 x 4.60 $\mathrm{mm}, \mathrm{MeOH}-1 \% \mathrm{NH}_{4} \mathrm{OH}$ in $\mathrm{H}_{2} \mathrm{O}, 1.0 \mathrm{~mL} / \mathrm{min}$, UV detection at $\left.254 \mathrm{~nm}, \mathrm{~T}_{\mathrm{R}}=14.5 \mathrm{~min}\right) \mathrm{NMR}$ and chromatographic properties matched those of authentic (-)-idiospermuline prepared earlier. ${ }^{7}$

\footnotetext{
${ }^{3}$ Verotta, L.; Peterlongo, F.; Elisabetsky, E.; Amador, T. A.; Nunes, D. S. J. Chromatogr. A. 1999, 841, $165-176$.

${ }^{4}$ Netto, C. A.; Siegfried, B.; Izquierdo, I. Behav. Neur. Biol. 1987, 48, 304-309.

${ }^{5}$ Amador, T. A.; Elisabetsky, E.; Souza, D. O. Neurochem. Res. 1996, 21, 97-102.

${ }^{6}$ Correa, C. R.; Kyle D. J.; Chakravarty, S.; Calixto, J.B. Brit .J. Pharmacol. 1996, 110, 552-558.

7 (a) Overman, L. E.; Peterson, E. A. Angew. Chem., Int. Ed. 2003, 42, 2525-2528. (b) Overman, L. E.; Peterson, E. A. Tetrahedron 2003, 59, 6905-6919.
} 
Part B.

Table 1. Effects of hodgkinsine and stereoisomers in the tail-flick and capsaicin models.

\begin{tabular}{|c|c|c|c|}
\hline Treatment & $\begin{array}{l}\text { Dose } \\
(\mathrm{mg} / \mathrm{Kg})\end{array}$ & $\begin{array}{l}\text { Tail flick } \\
\text { (sec) }\end{array}$ & $\begin{array}{l}\text { Capsaicin } \\
\text { (licking sec) }\end{array}$ \\
\hline Saline & & $0.76 \pm 0.3$ & $60.4 \pm 5.7$ \\
\hline DMSO & & $0.52 \pm 0.3$ & $60.7 \pm 4.8$ \\
\hline Morphine & & $5.74 \pm 0.3^{b}$ & \\
\hline MK-801 & & & $3.3 \pm 0.9^{b}$ \\
\hline \multirow[t]{2}{*}{ Hodgkinsine isolated } & 2.5 & & $39.5 \pm 7.9^{b}$ \\
\hline & 5.0 & $2.19 \pm 0.6^{\mathrm{a}}$ & \\
\hline \multirow{2}{*}{$\begin{array}{l}\text { Hodgkinsine isolated } \\
\text { (salt) }\end{array}$} & 2.5 & & $37.9 \pm 4.8^{b}$ \\
\hline & 5.0 & $3.92 \pm 0.7^{b}$ & \\
\hline \multirow[t]{4}{*}{ Hodgkinsine synthetic $\mathbf{1}$} & 0.5 & & $35.7 \pm 7.9^{b}$ \\
\hline & 2.5 & & $36.4 \pm 5.1^{\mathrm{b}}$ \\
\hline & 5.0 & $2.26 \pm 0.8^{\mathrm{a}}$ & \\
\hline & 10.0 & $2.40 \pm 0.8^{\mathrm{a}}$ & \\
\hline \multirow{2}{*}{$\begin{array}{l}\text { Hodgkinsine synthetic } \mathbf{1} \\
\text { (salt) }\end{array}$} & 2.5 & & $45.2 \pm 3.7^{\mathrm{a}}$ \\
\hline & 5.0 & $2.43 \pm 0.4^{\mathrm{b}}$ & \\
\hline \multirow{4}{*}{ ent-1 } & 0.5 & & $43.4 \pm 9.4$ \\
\hline & 2.5 & & $48.8 \pm 10.5$ \\
\hline & 5.0 & $2.13 \pm 0.5$ & \\
\hline & 10.0 & $2.94 \pm 0.7^{b}$ & \\
\hline \multirow{4}{*}{2} & 0.5 & & $47.9 \pm 5.6$ \\
\hline & 2.5 & & $39.3 \pm 8.2^{\mathrm{a}}$ \\
\hline & 5.0 & $0.53 \pm 0.2$ & $52.7 \pm 5.3$ \\
\hline & 10.0 & $2.13 \pm 0.5^{\mathrm{a}}$ & \\
\hline \multirow{4}{*}{ ent-2 } & 0.5 & & $43.3 \pm 8.7$ \\
\hline & 2.5 & & $38.3 \pm 10.0^{\mathrm{a}}$ \\
\hline & 5.0 & $2.85 \pm 0.9^{\mathrm{a}}$ & $19.3 \pm 6.2^{b}$ \\
\hline & 10.0 & $3.82 \pm 0.4^{\mathrm{b}}$ & \\
\hline \multirow{4}{*}{3} & 0.5 & & $55.4 \pm 3.8$ \\
\hline & 2.5 & & $41.3 \pm 3.7^{\mathrm{a}}$ \\
\hline & 5.0 & $1.84 \pm 0.5^{\mathrm{a}}$ & $26.5 \pm 6.5^{b}$ \\
\hline & 10.0 & $2.50 \pm 0.9^{\mathrm{a}}$ & \\
\hline \multirow[t]{2}{*}{ ent-3 } & 0.5 & - & $44.6 \pm 8.9$ \\
\hline & 2.5 & - & $43.7 \pm 8.4$ \\
\hline \multirow{4}{*}{4} & 0.5 & & $54.1 \pm 4.3$ \\
\hline & 2.5 & & $42.7 \pm 6.2$ \\
\hline & 5.0 & $0.99 \pm 0.9$ & $33.6 \pm 5.9^{b}$ \\
\hline & 10.0 & $1.82 \pm 1.0$ & \\
\hline \multirow{4}{*}{ ent-4 } & 0.5 & & $62.2 \pm 7.2$ \\
\hline & 2.5 & & $47.2 \pm 9.0$ \\
\hline & 5.0 & $0.73 \pm 0.4$ & $41.2 \pm 6.5$ \\
\hline & 10.0 & $1.19 \pm 0.5$ & \\
\hline 5 & 0.5 & & $56.9 \pm 5.0$ \\
\hline
\end{tabular}




\begin{tabular}{l|l|l|l}
\hline \multicolumn{1}{l|}{} & 2.5 & & $25.8 \pm 3.6^{\mathrm{b}}$ \\
& 5.0 & $0.28 \pm 0.6$ & \\
& 10.0 & $2.30 \pm 0.9$ & \\
\hline \multirow{3}{*}{6} & 0.5 & & $40.8 \pm 6.7^{\mathrm{a}}$ \\
& 2.5 & & $52.5 \pm 8.7$ \\
& 5.0 & $1.34 \pm 0.6$ & \\
\hline
\end{tabular}

Data represent as mean \pm SEM. ${ }^{a}=p<0,05,{ }^{b} p<0,01$ vs. vehicle. Tail flick sec refer to delta (in secs) from post and pre drug measures.. $\mathrm{N}=5-10$ for test groups.

Table 2: Reversibility of hodgkinsine derivatives effects on tail flick by Naloxone (15mg/kg).

\begin{tabular}{l|l|c}
\hline Treatment & $\begin{array}{l}\text { Dose } \\
\text { (mg/Kg) }\end{array}$ & $\begin{array}{c}\text { Tail } \\
\text { Flick } \\
\text { (sec) }\end{array}$ \\
\hline Saline & - & $0.76 \pm 0.32$ \\
Morphine & 6.0 & $\begin{array}{c}5.74 \pm 0.3 \\
\text { b,c }\end{array}$ \\
NAL +Morphine & 6.0 & $0.93 \pm 0.3$ \\
DMSO & - & $0.52 \pm 0.3$ \\
\hline Hodgkinsine synthetic 1 & 5.0 & $2.26 \pm 0.8^{\mathrm{a}}$ \\
NAL + Hodgkinsine synthetic 1 & 5.0 & $1.13 \pm 0.9^{\mathrm{a}}$ \\
\hline $\mathbf{2}$ & 10.0 & $2.13 \pm 0.5^{\mathrm{a}}$ \\
NAL $+\mathbf{2}$ & 10.0 & $0.67 \pm 0.9$ \\
\hline ent-2 & 10.0 & $3.82 \pm 0.4^{\mathrm{b}}$ \\
NAL + ent-2 & 10.0 & $2.93 \pm 0.8^{\mathrm{b}}$ \\
\hline $\mathbf{3}$ & 10.0 & $2.50 \pm 0.9^{\mathrm{a}}$ \\
NAL $+\mathbf{3}$ & 10.0 & $3.01 \pm 0.6^{\mathrm{b}}$ \\
\hline $\mathbf{6}$ & 10.0 & $3.22 \pm 0.7^{\mathrm{b}}$ \\
NAL $+\mathbf{6}$ & 10.0 & $2.24 \pm 0.6^{\mathrm{b}}$ \\
\hline
\end{tabular}

Data represent as mean \pm SEM, refer to delta (in secs) from post and pre drug measures. NAL $=$ Naloxone. ${ }^{a} p<0.05{ }^{b} p<0.01$ vs. vehicle, ${ }^{c} p<0.01$ vs. same group pre-treated with naloxone. $\mathrm{N}=5-10$ in test groups. 
Part C.

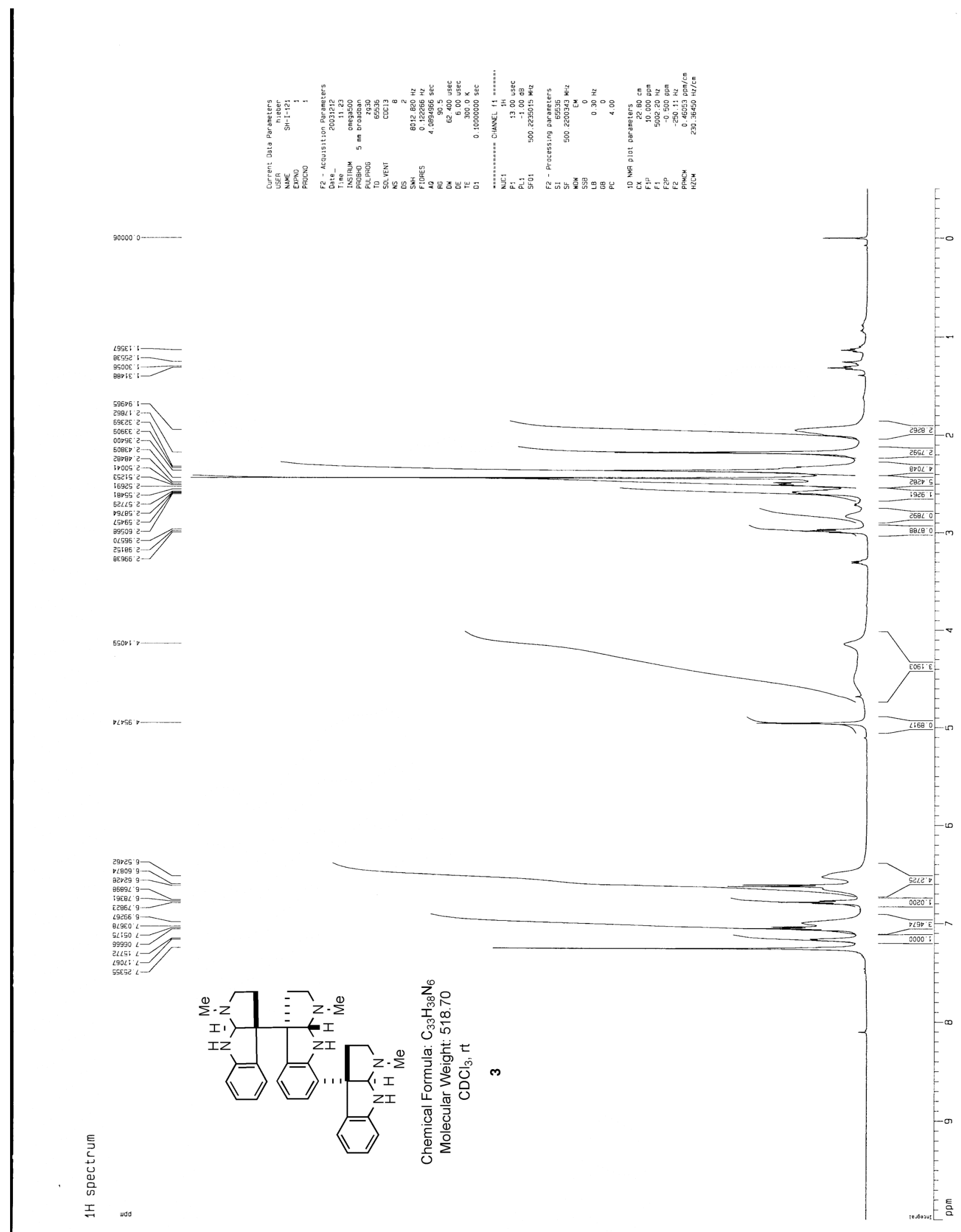




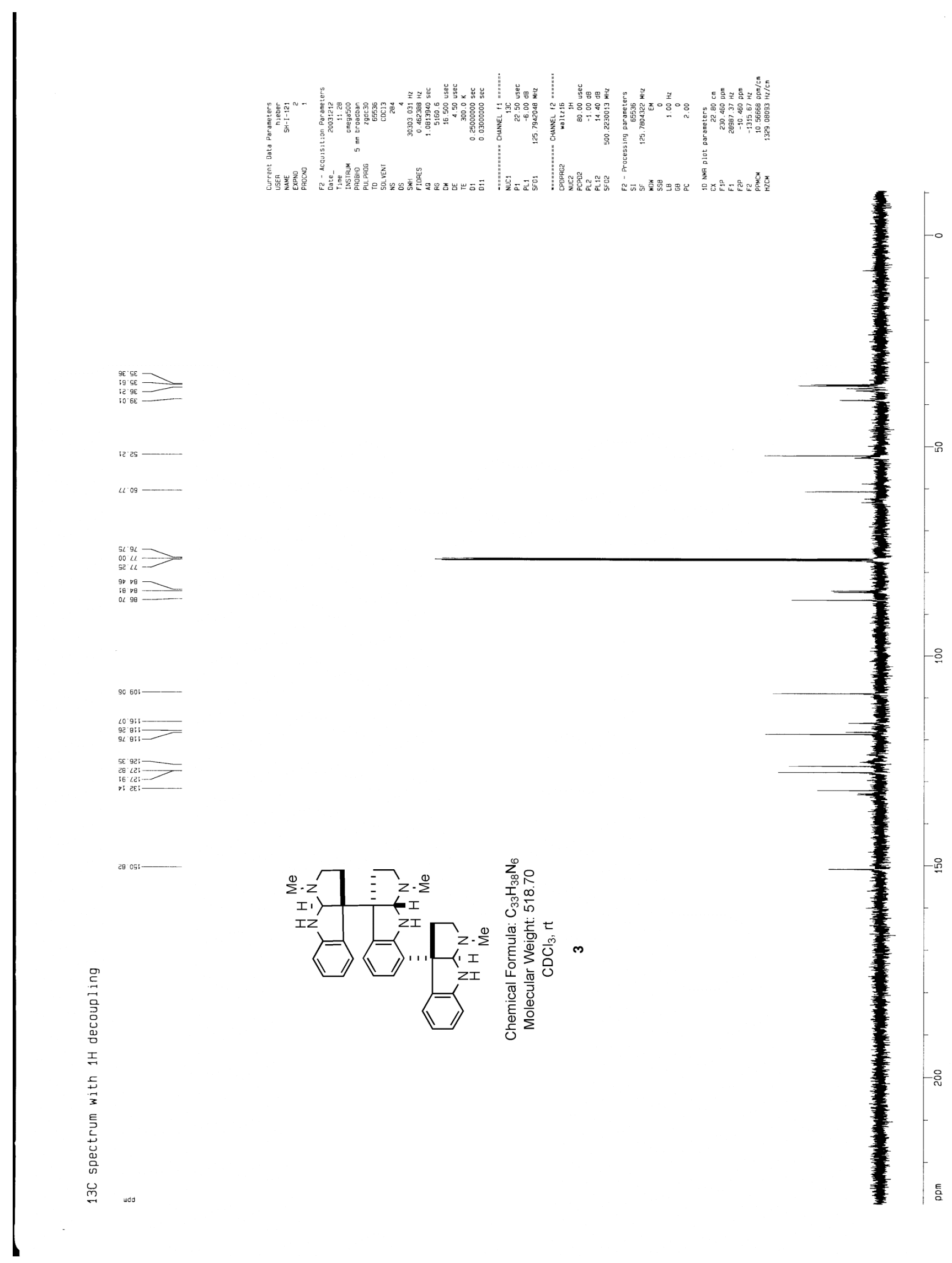




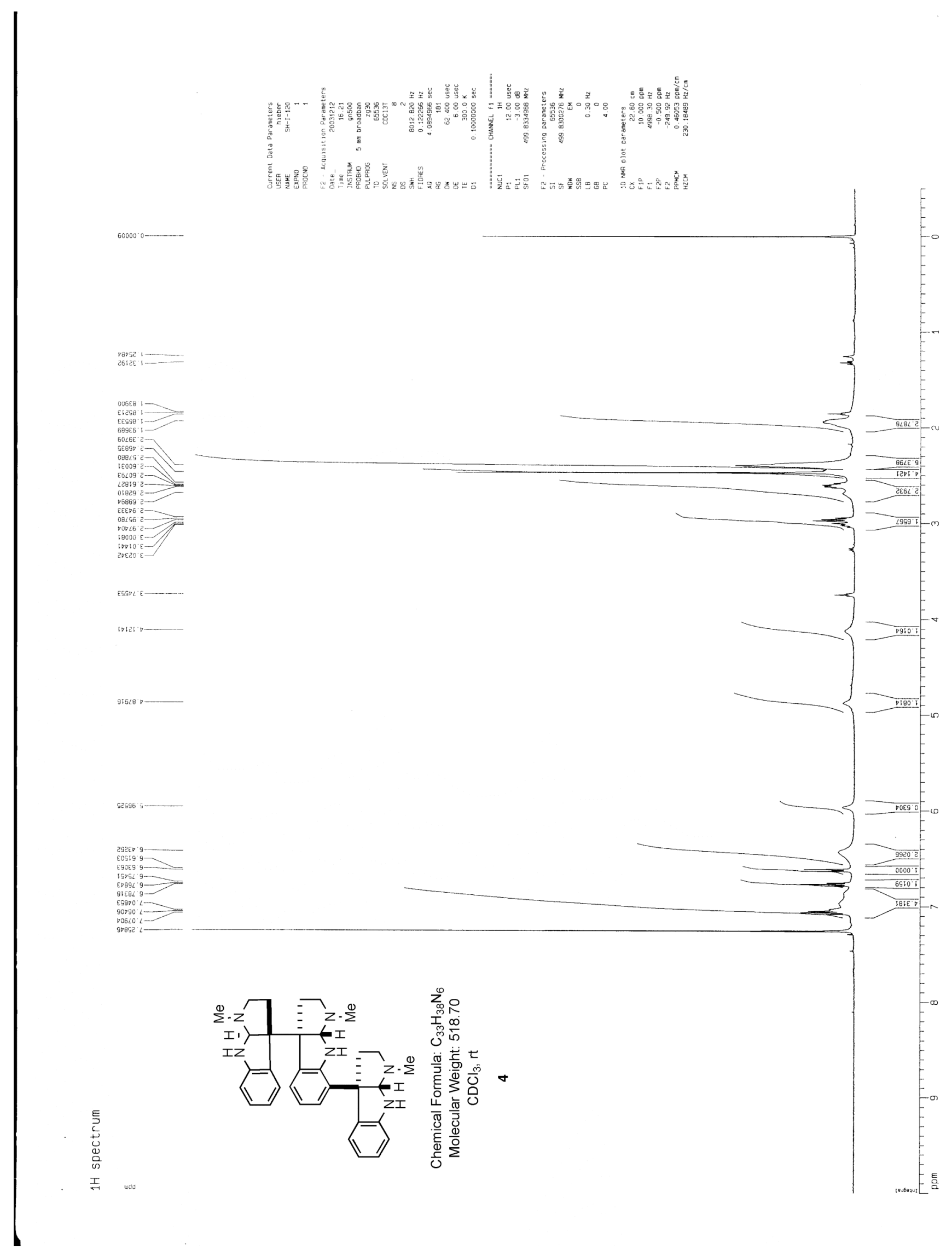




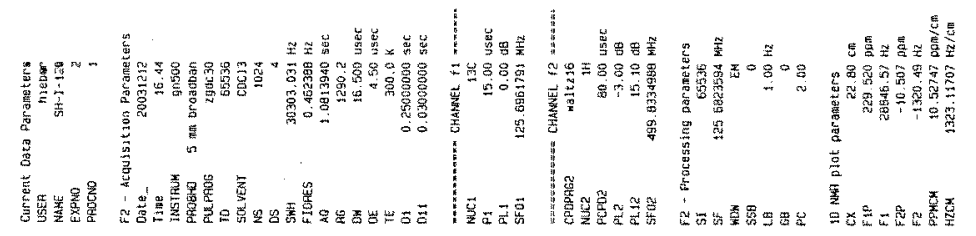

(4)
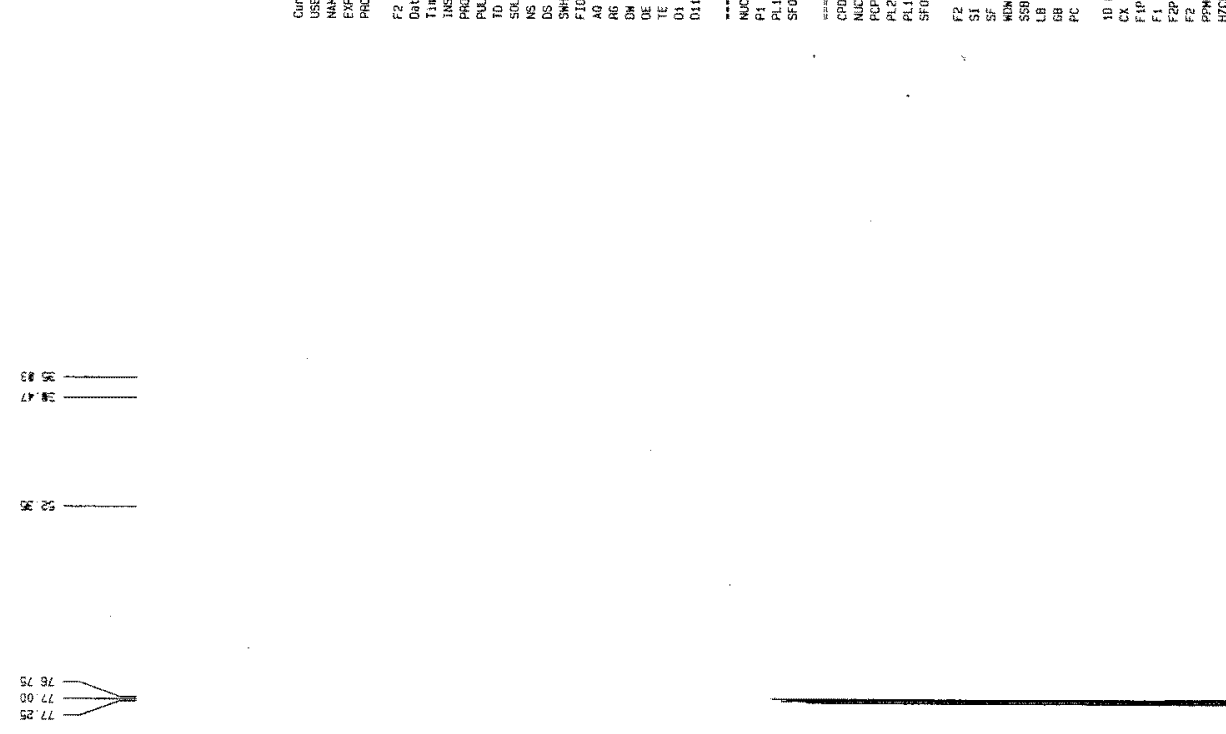

II:

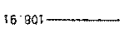

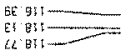

81 $22 x-$

39.065 -

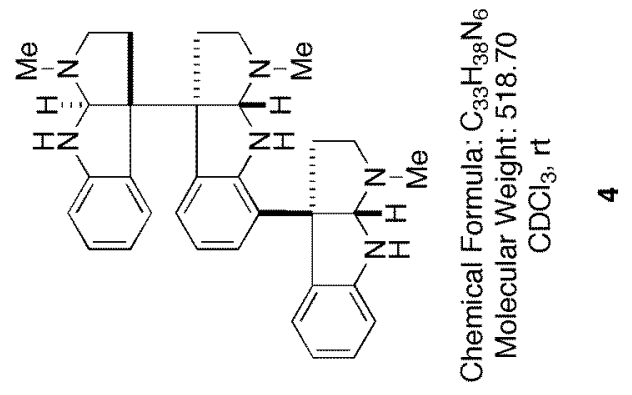




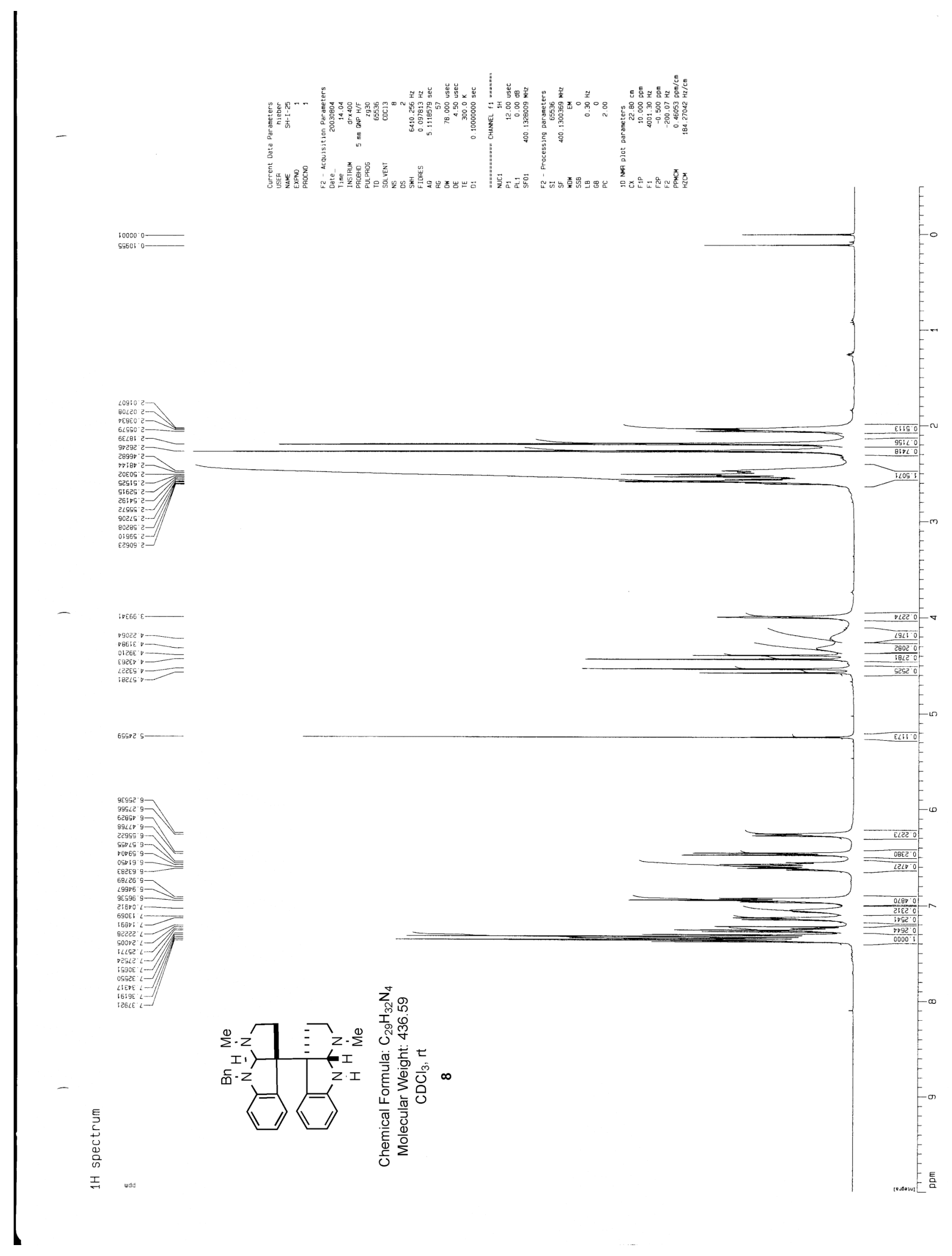




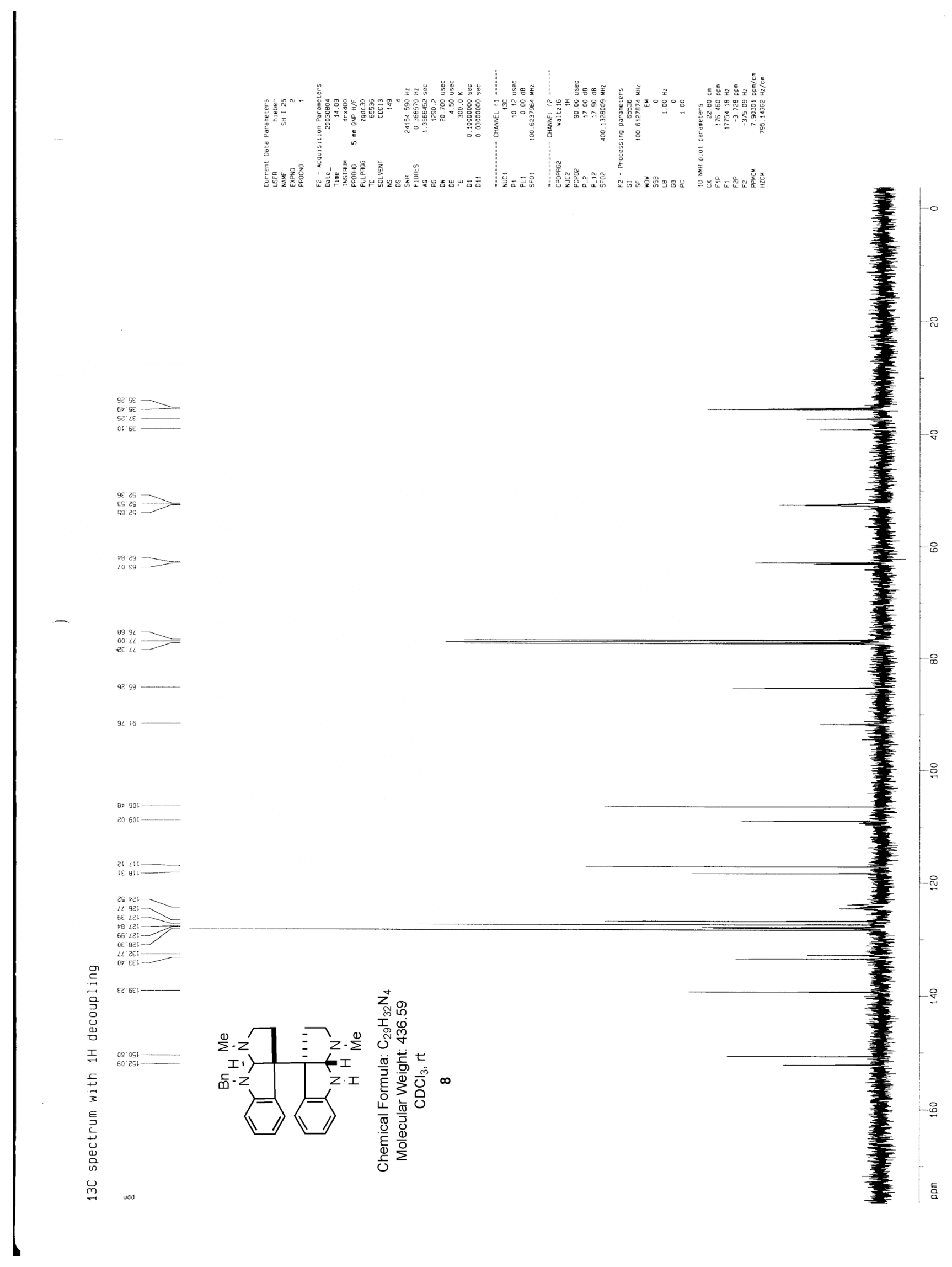




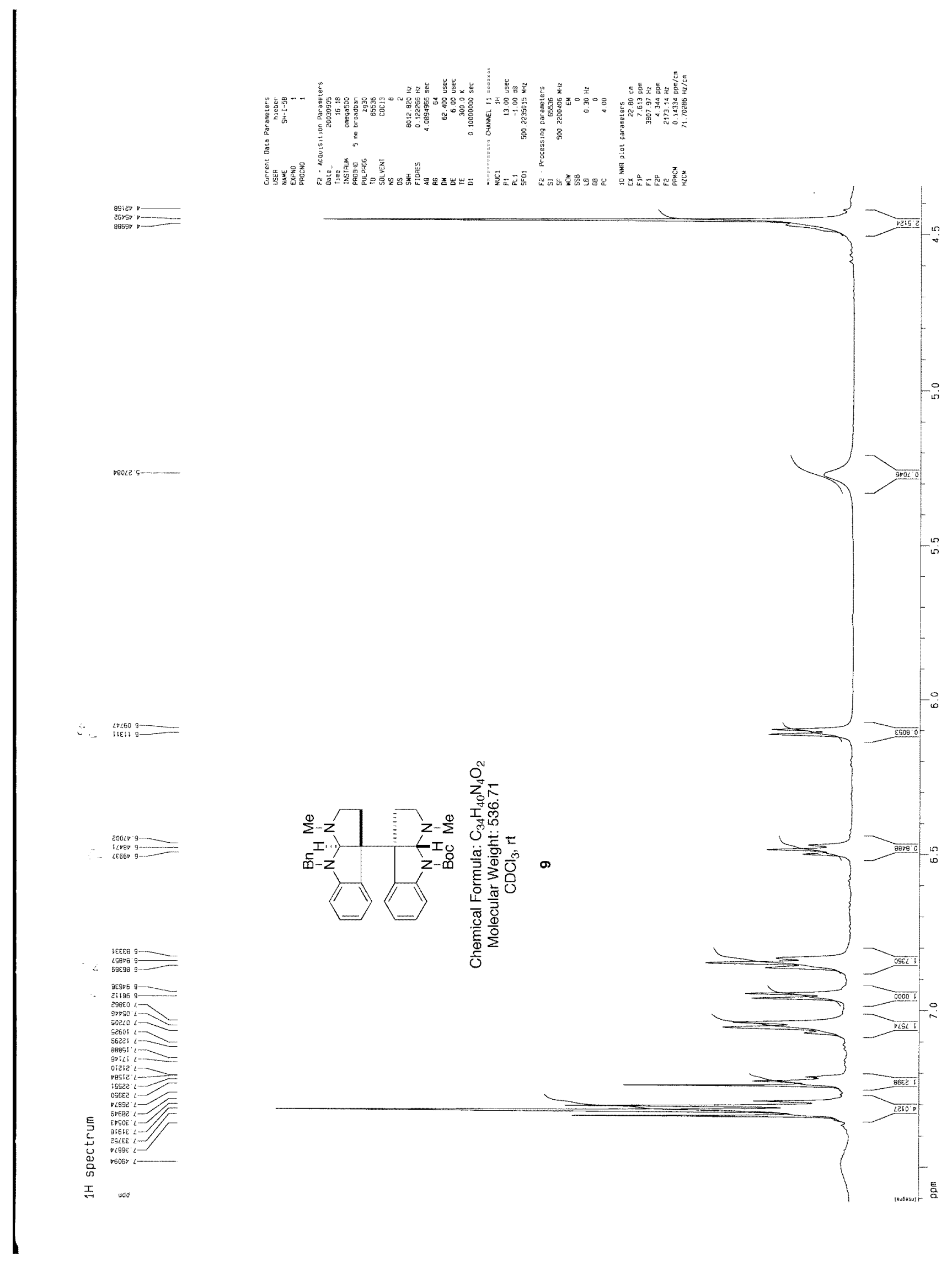




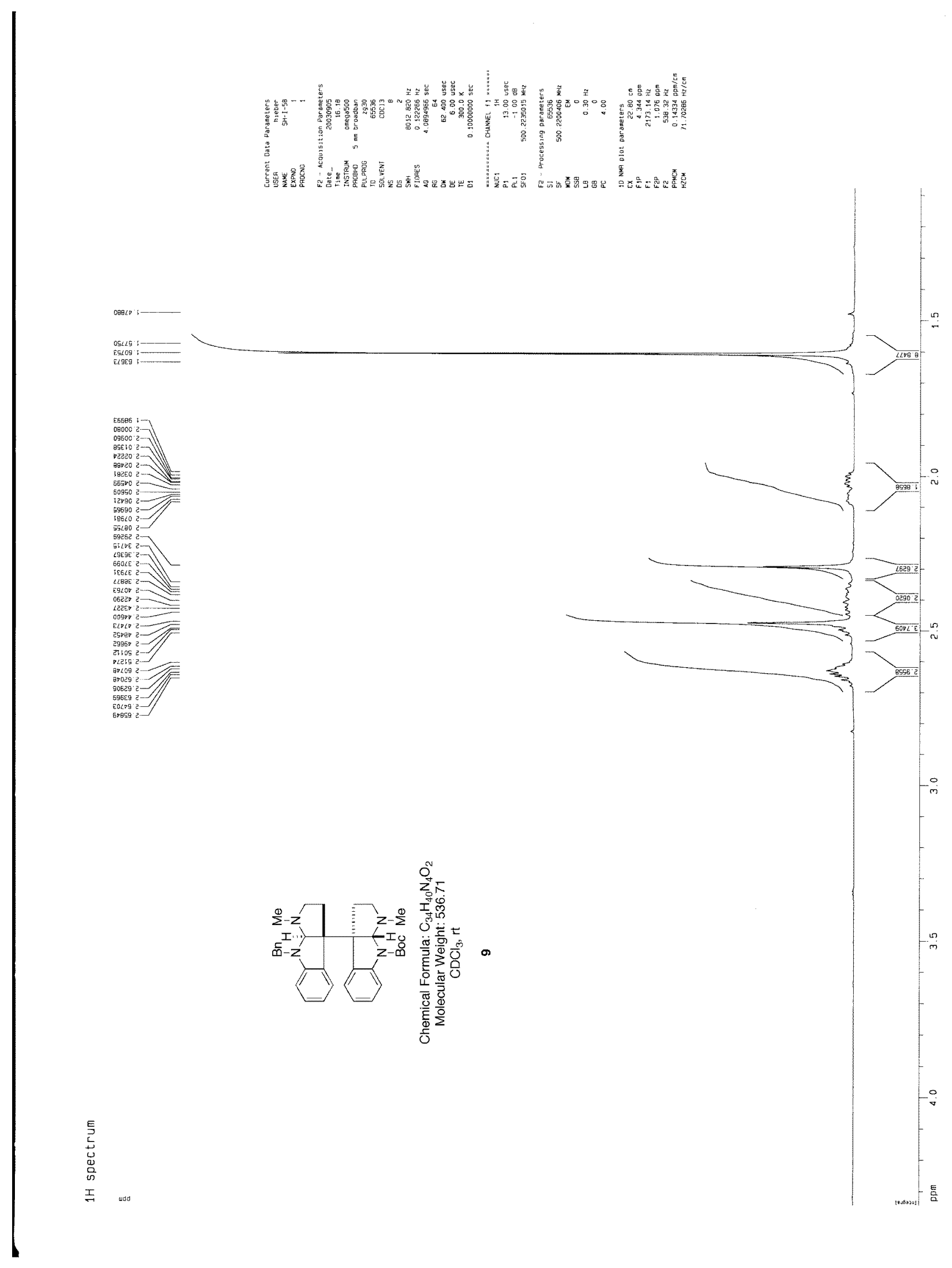




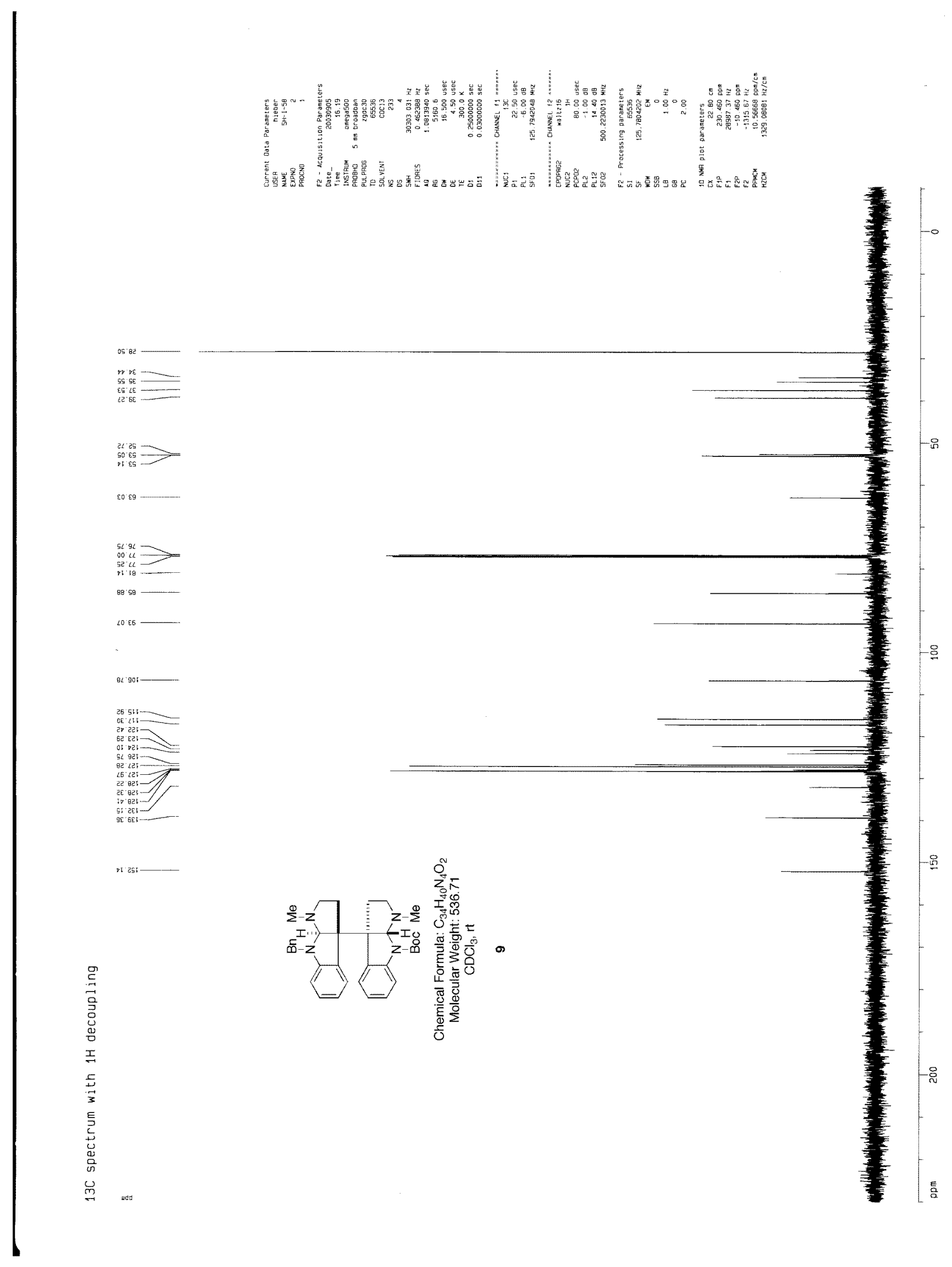




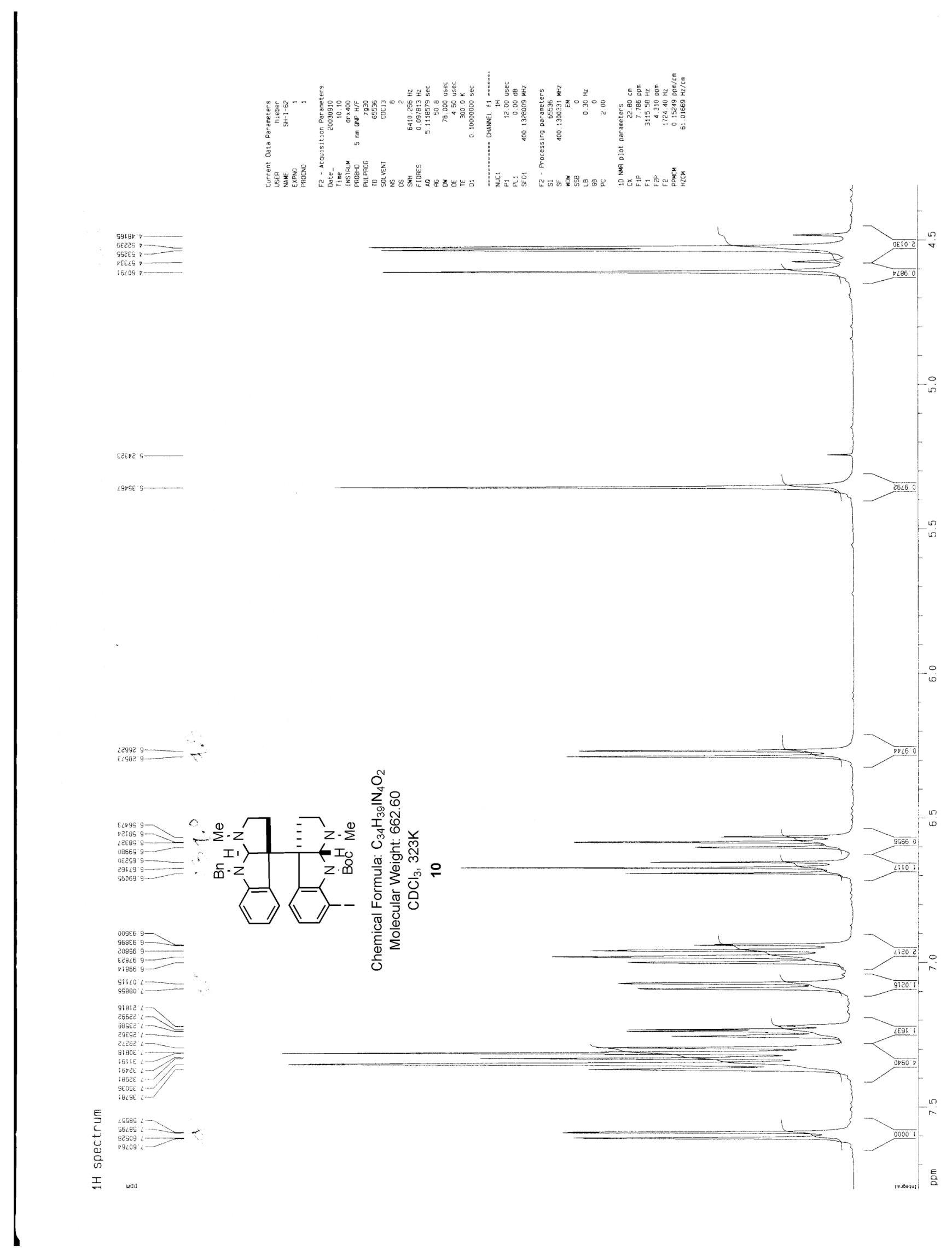




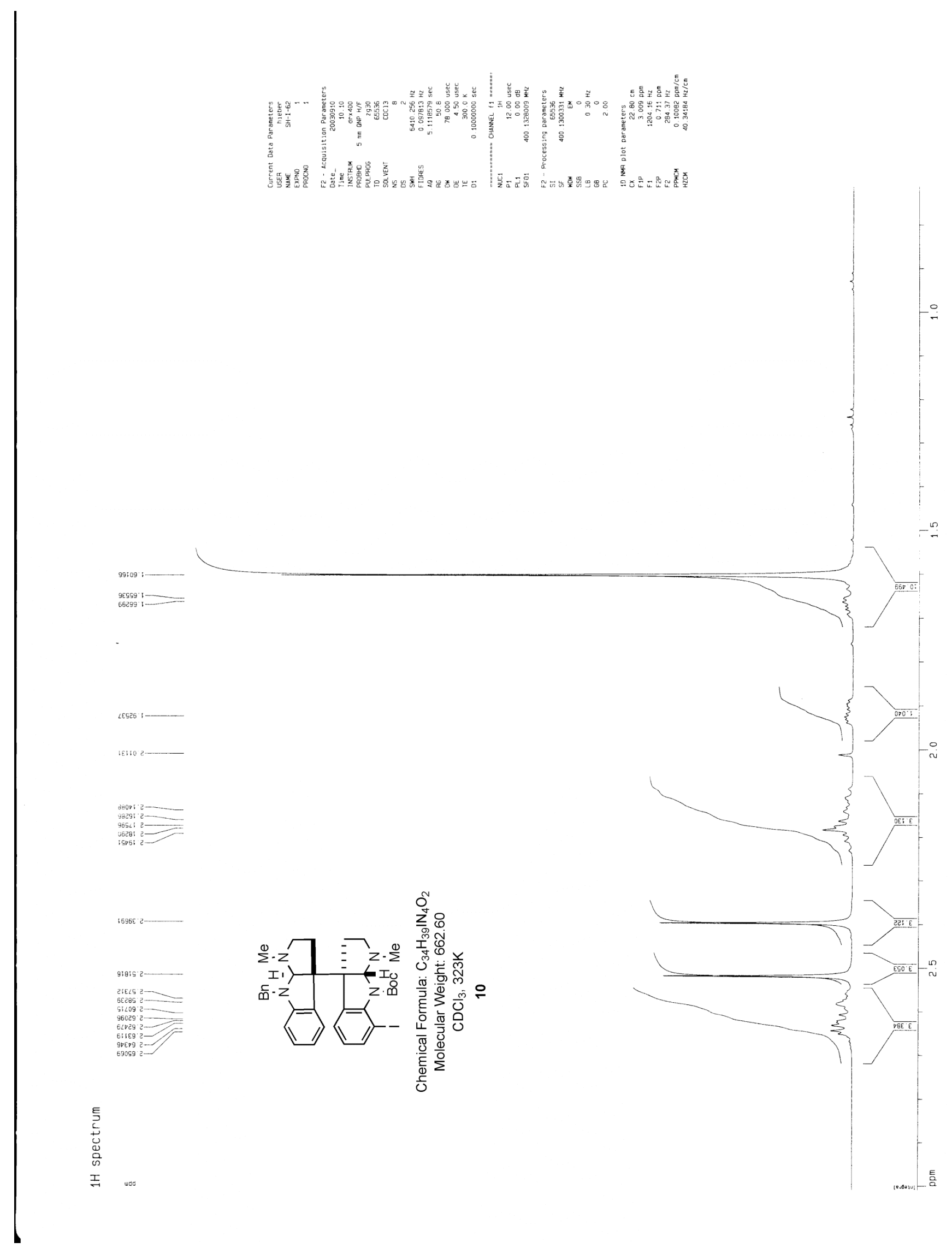




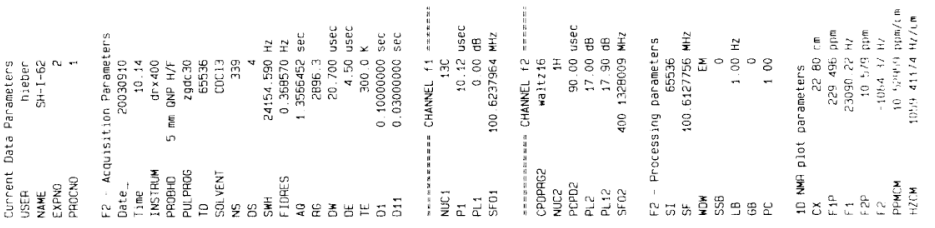

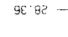

还

02
69
$65=\square$

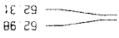

${ }_{00}^{90} 46$

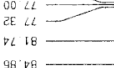

96 b8

os

DL 26

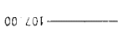

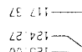

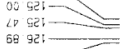

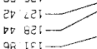

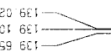

${ }^{3}<30$

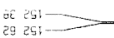

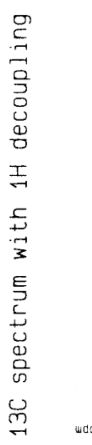

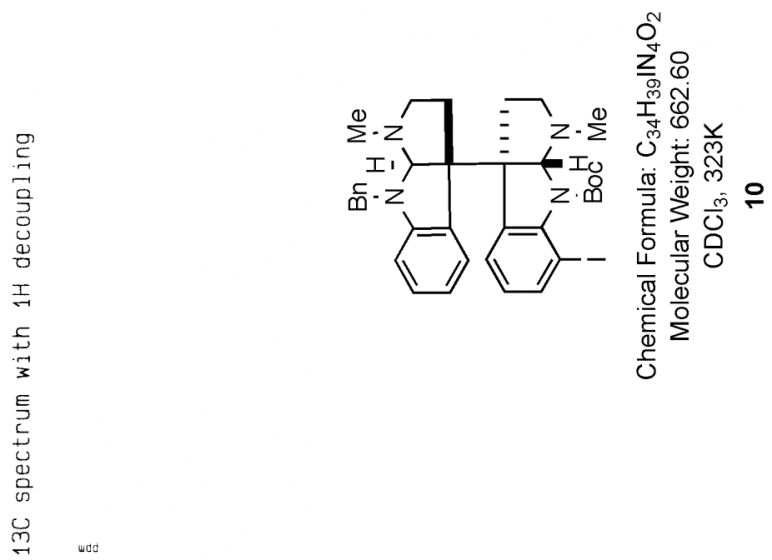




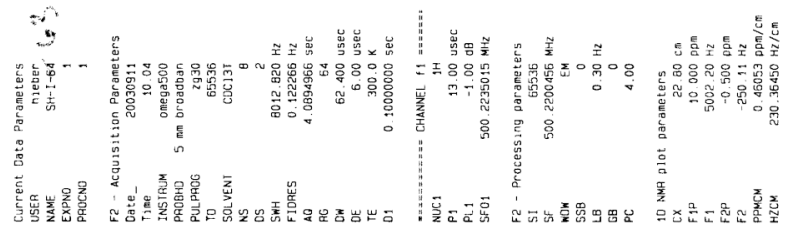

02000.0

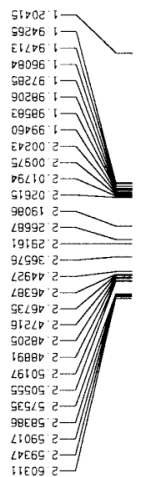

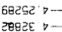

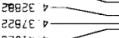

I9615 $5=$

$\angle 5662 \cdot 9-$
$59608,9-1$

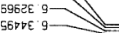

$\angle 95 \angle 9)^{2}>$

6веv 9

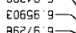

868669

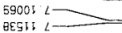

${ }_{565522}^{8662} 2-$

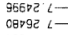

政:

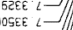

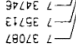

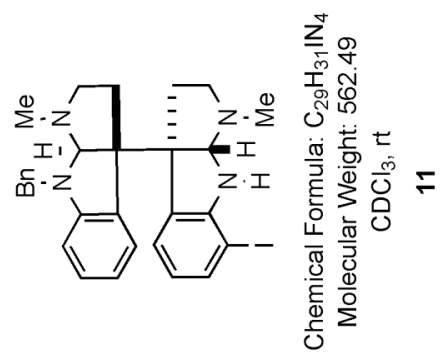

$$
-\infty
$$

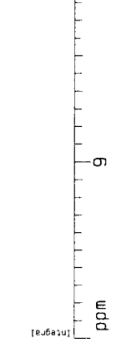




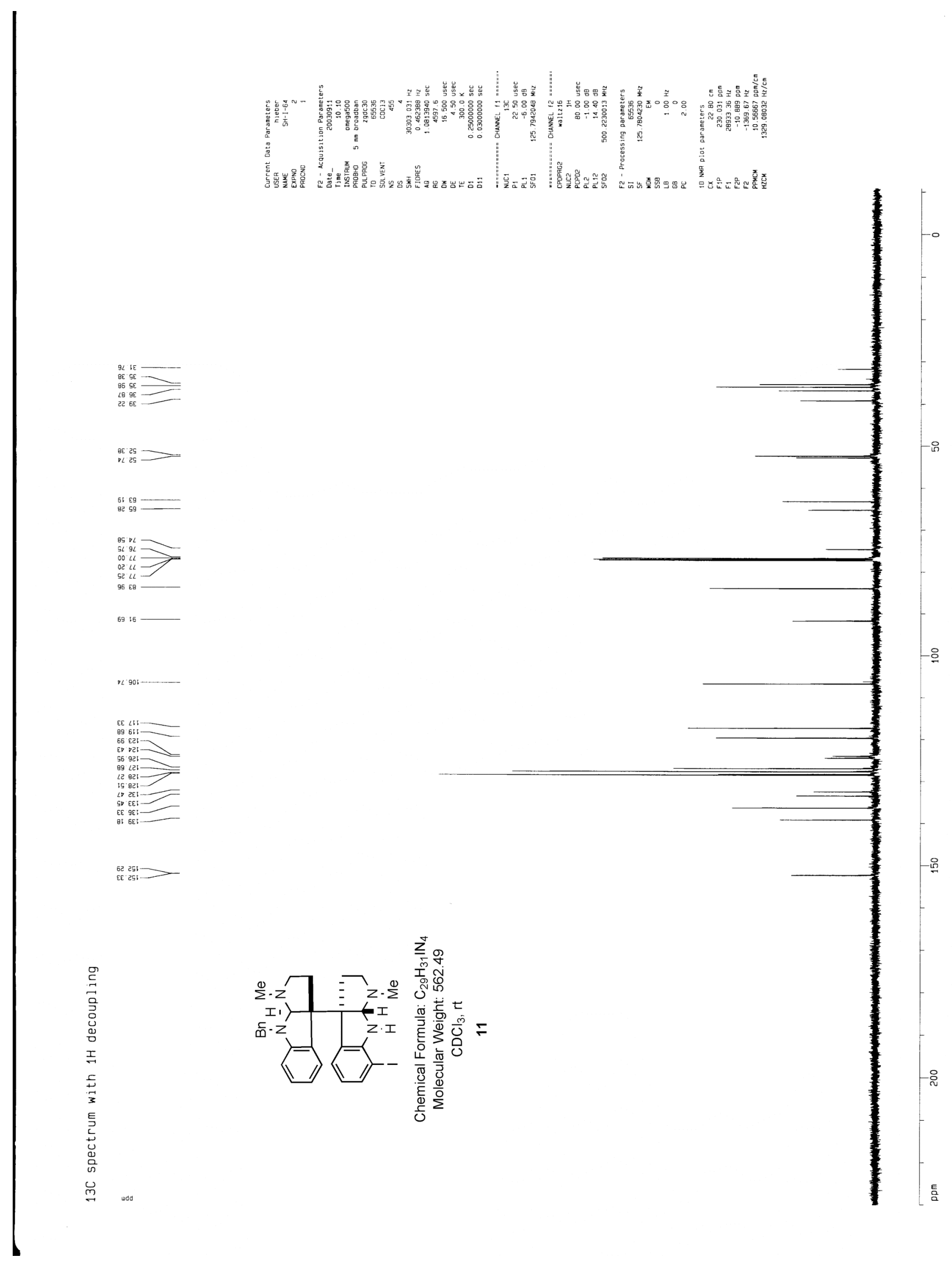




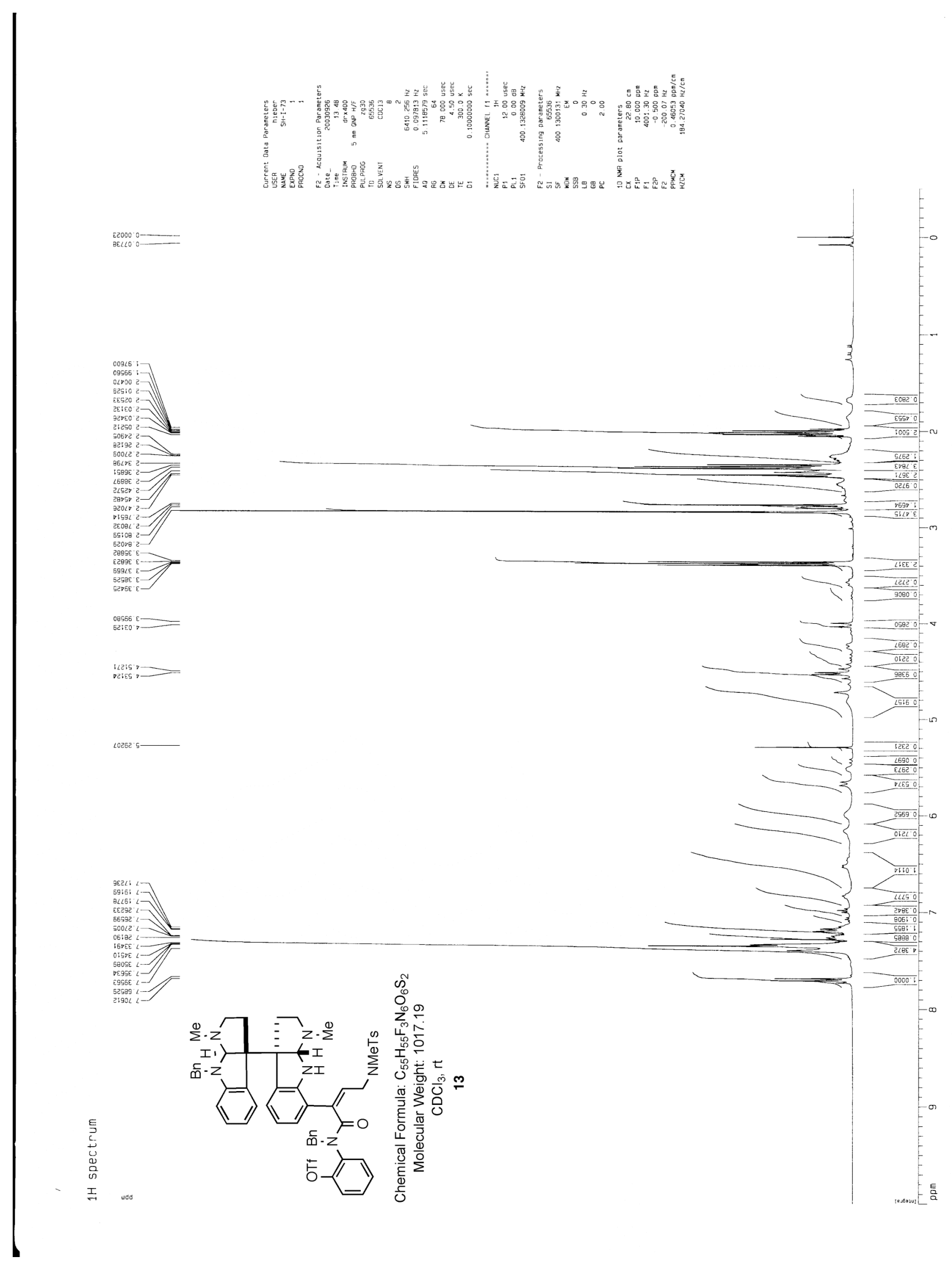



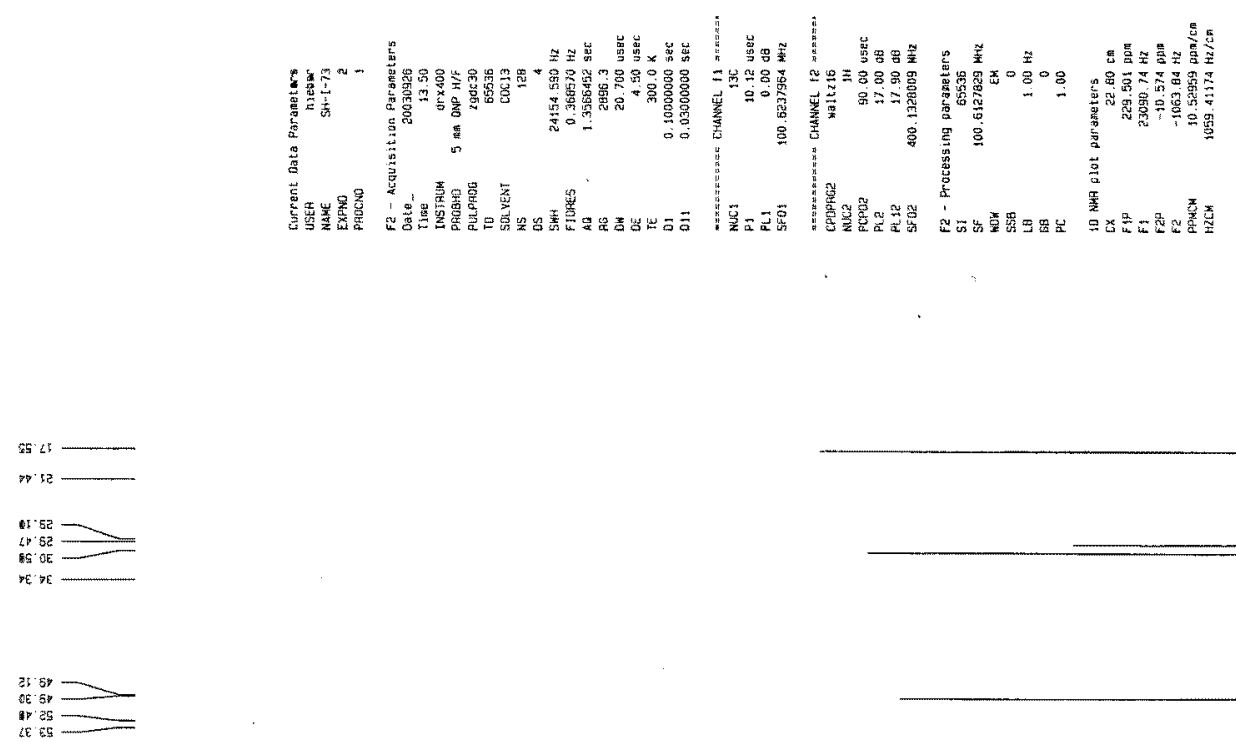

at

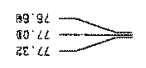
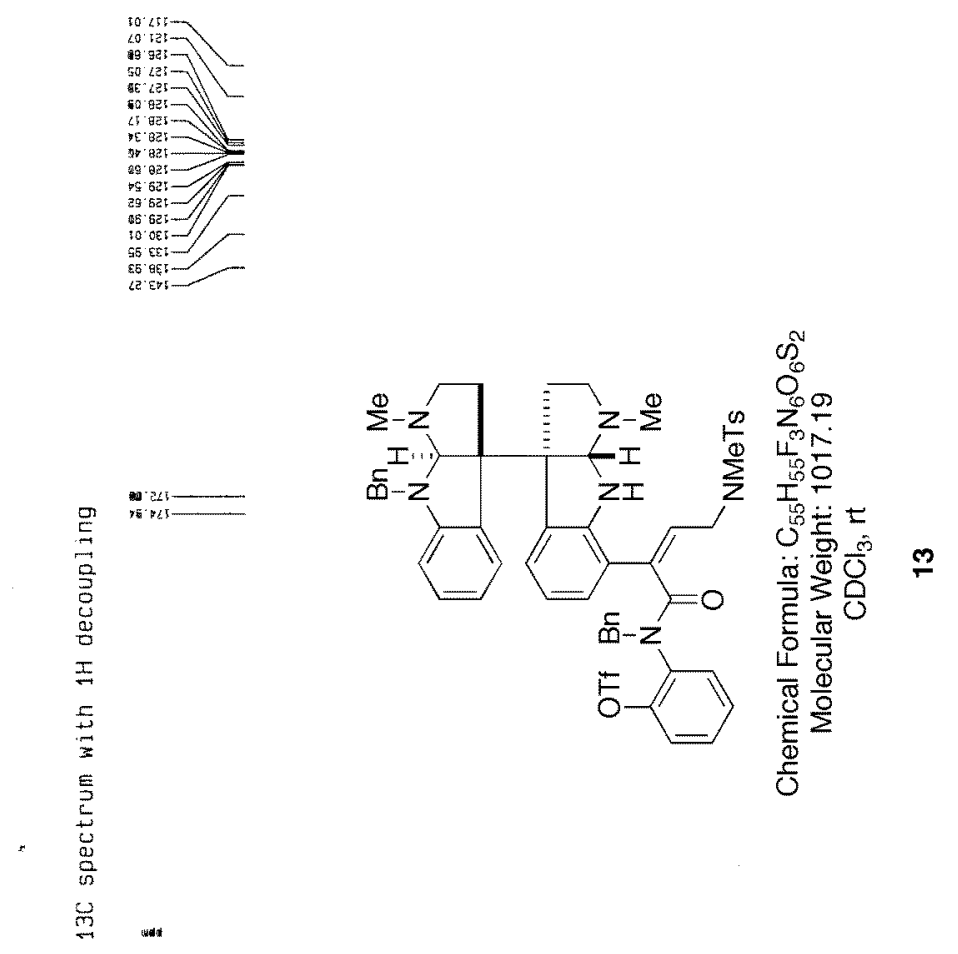


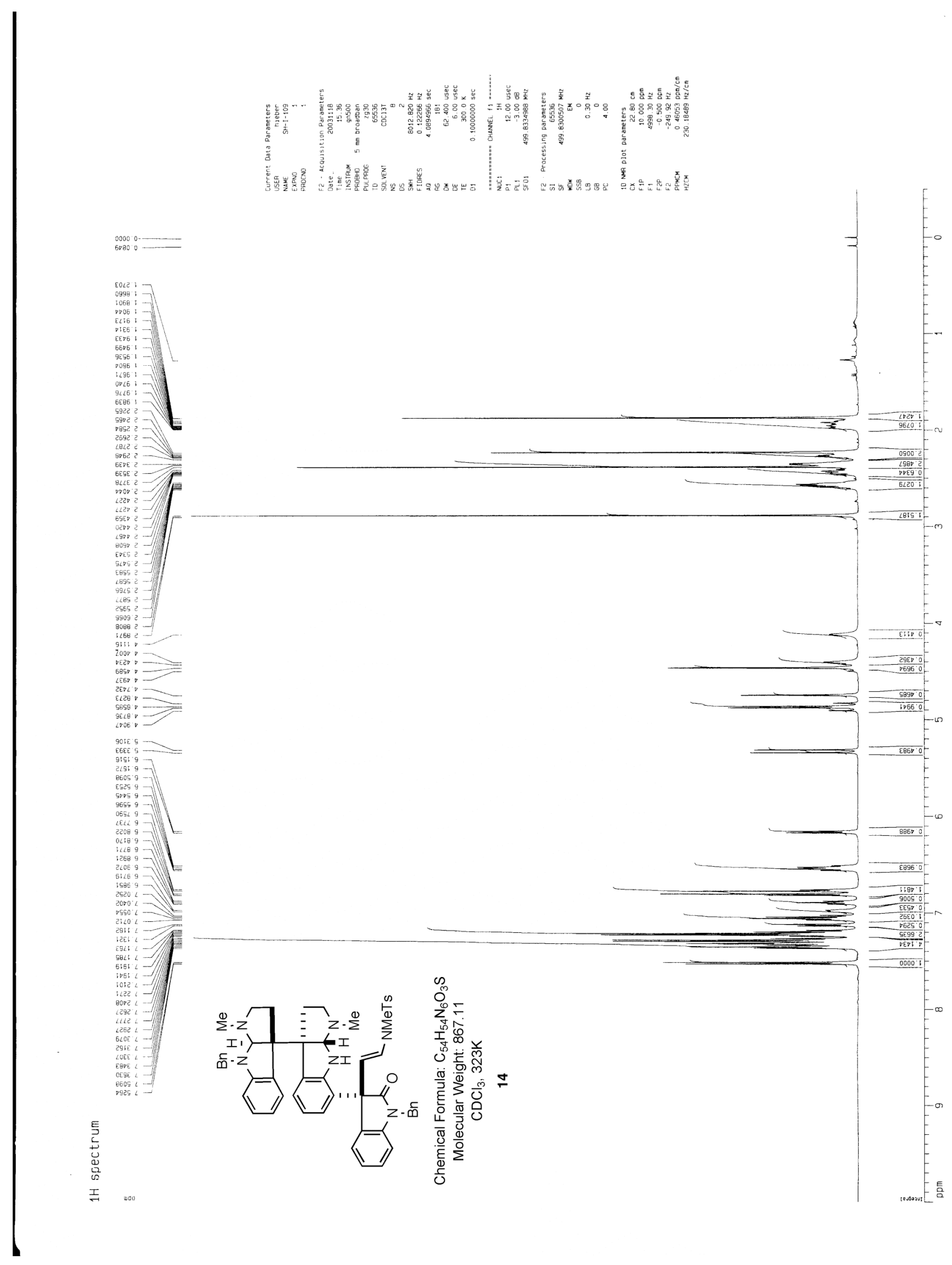




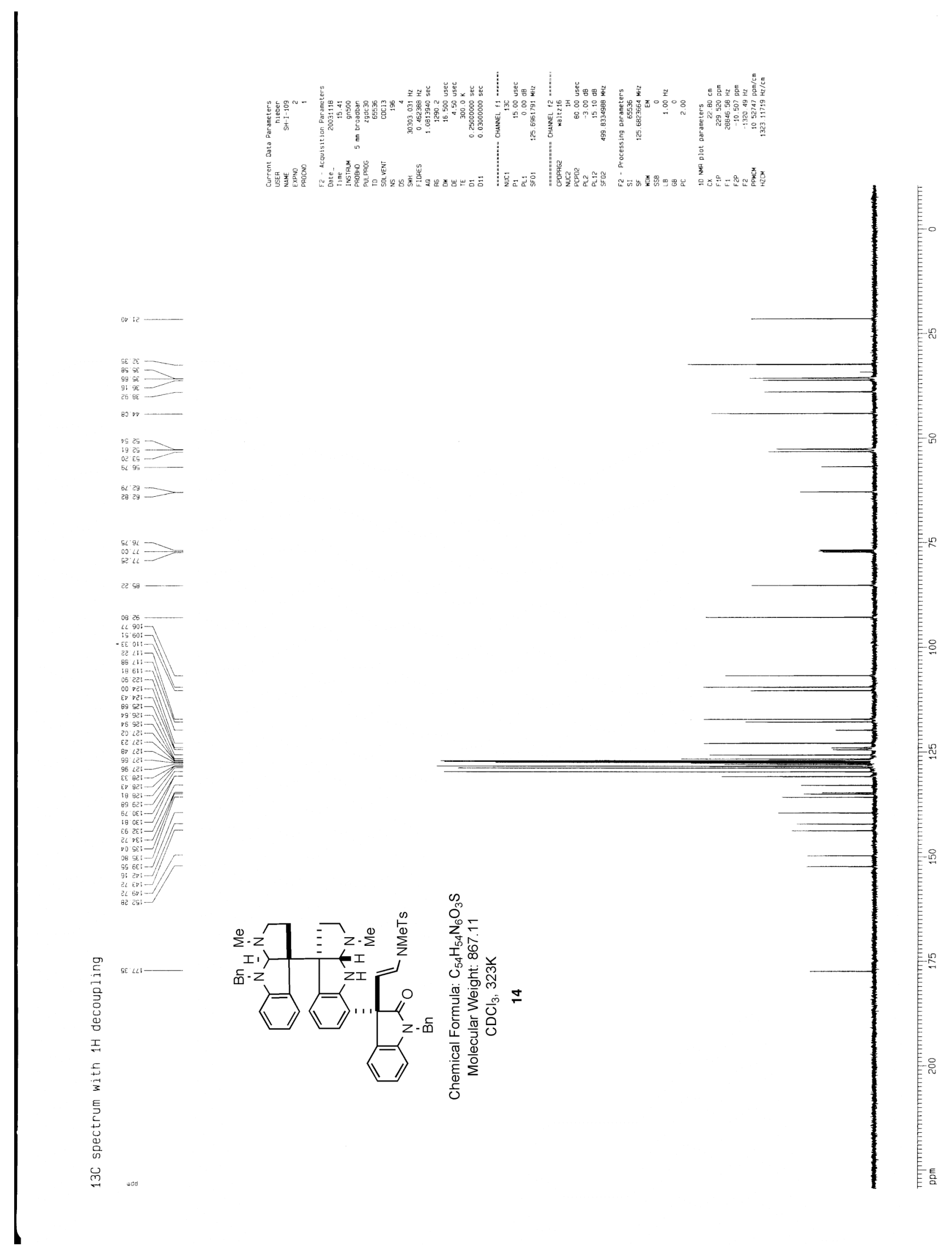




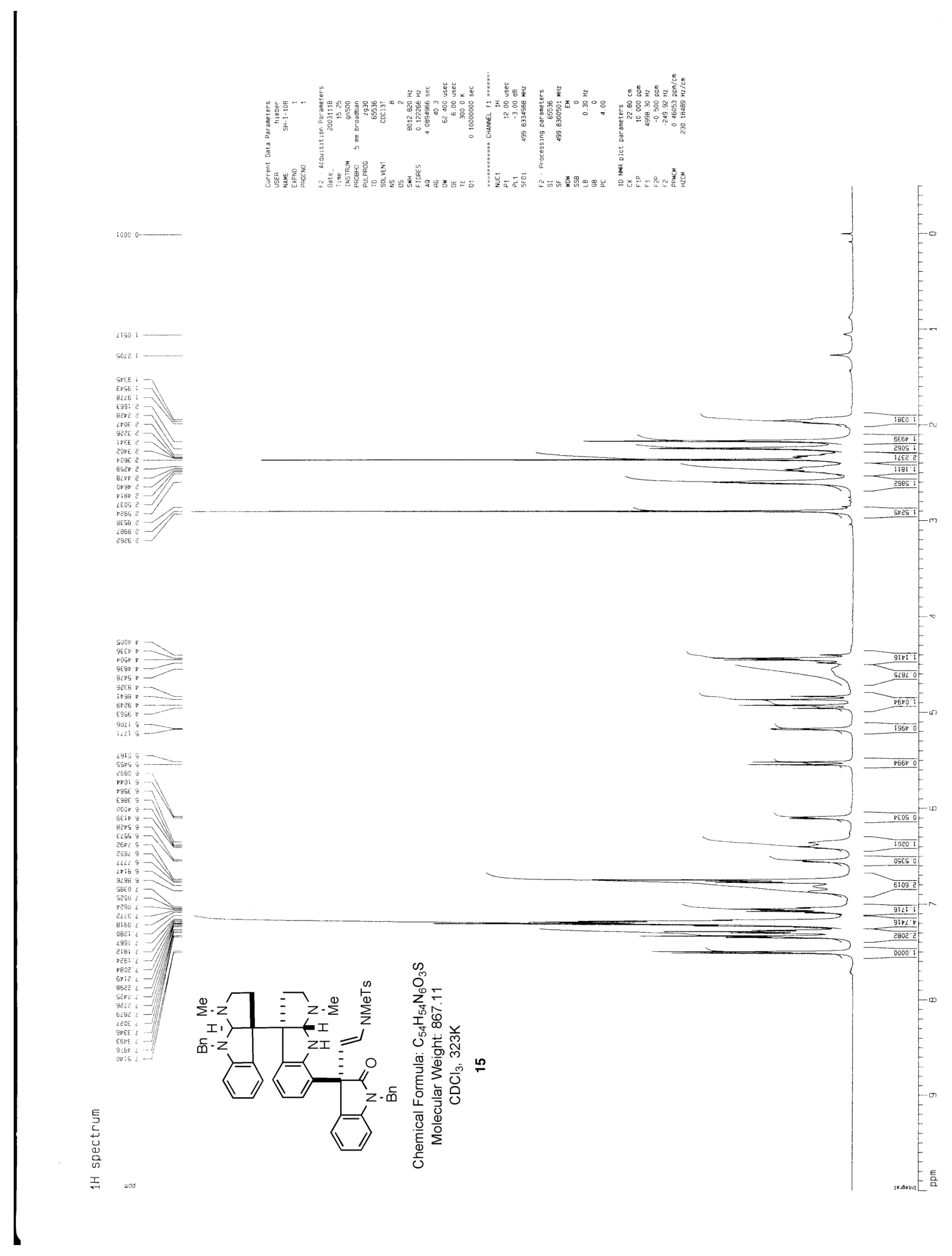




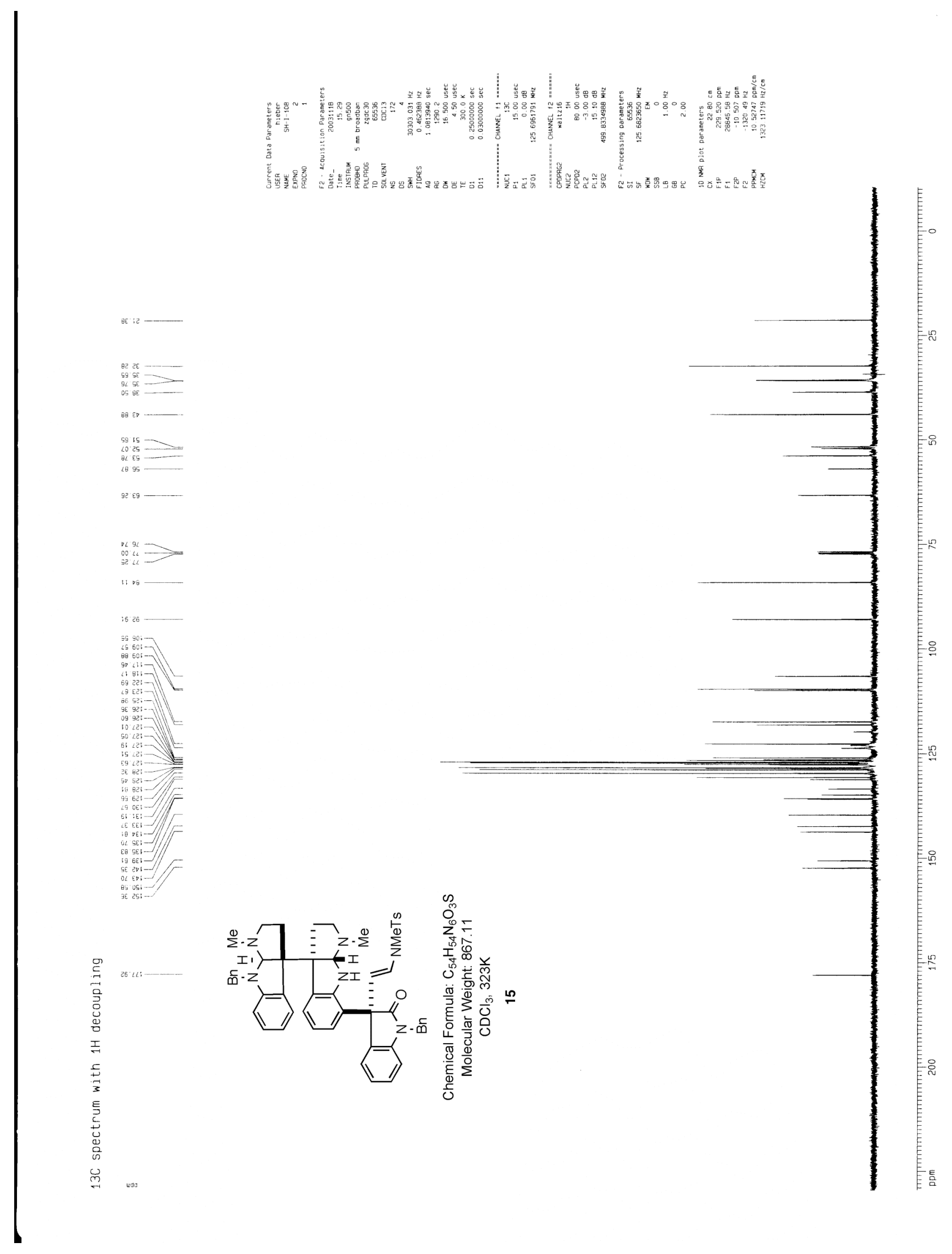



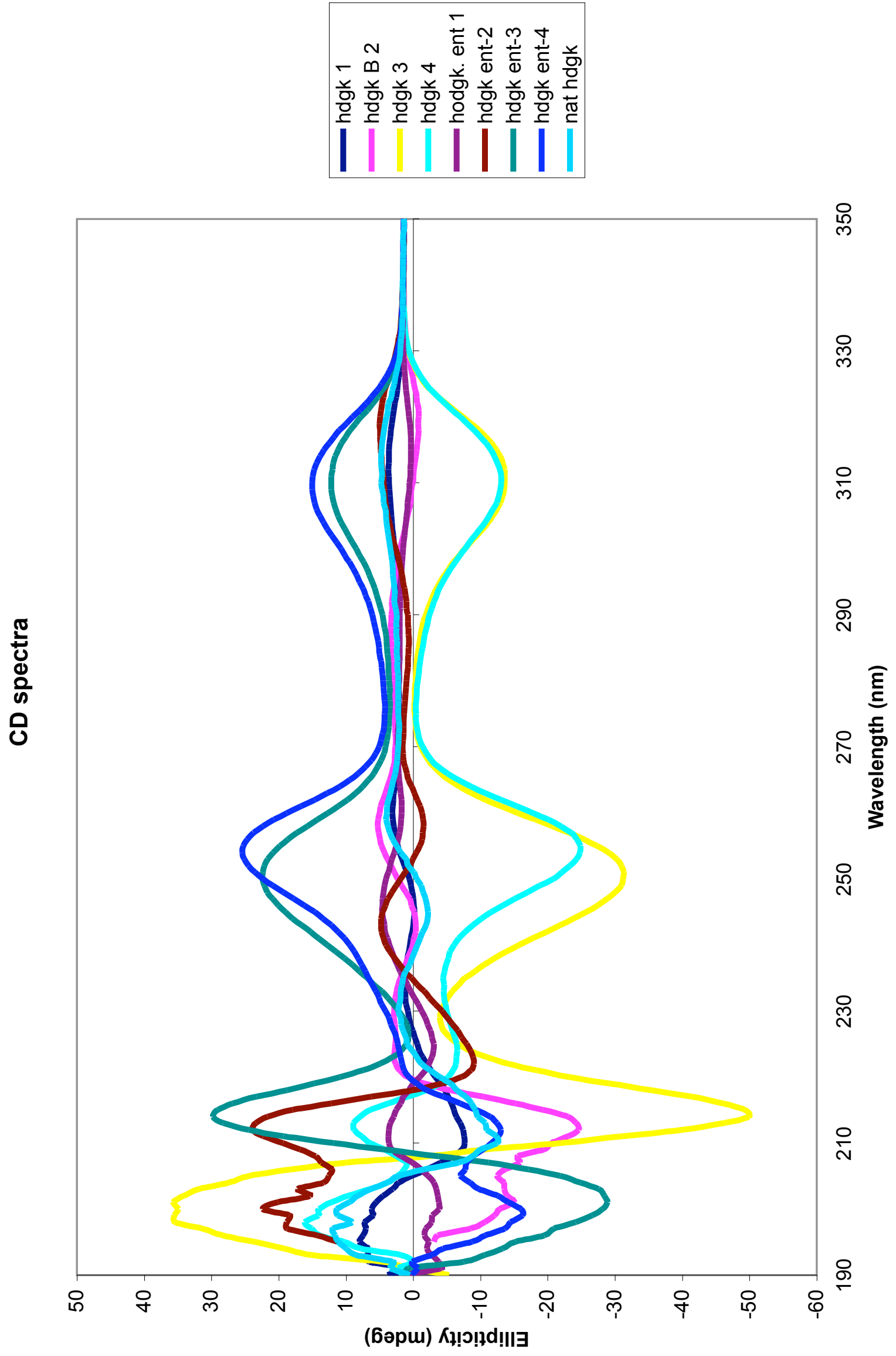


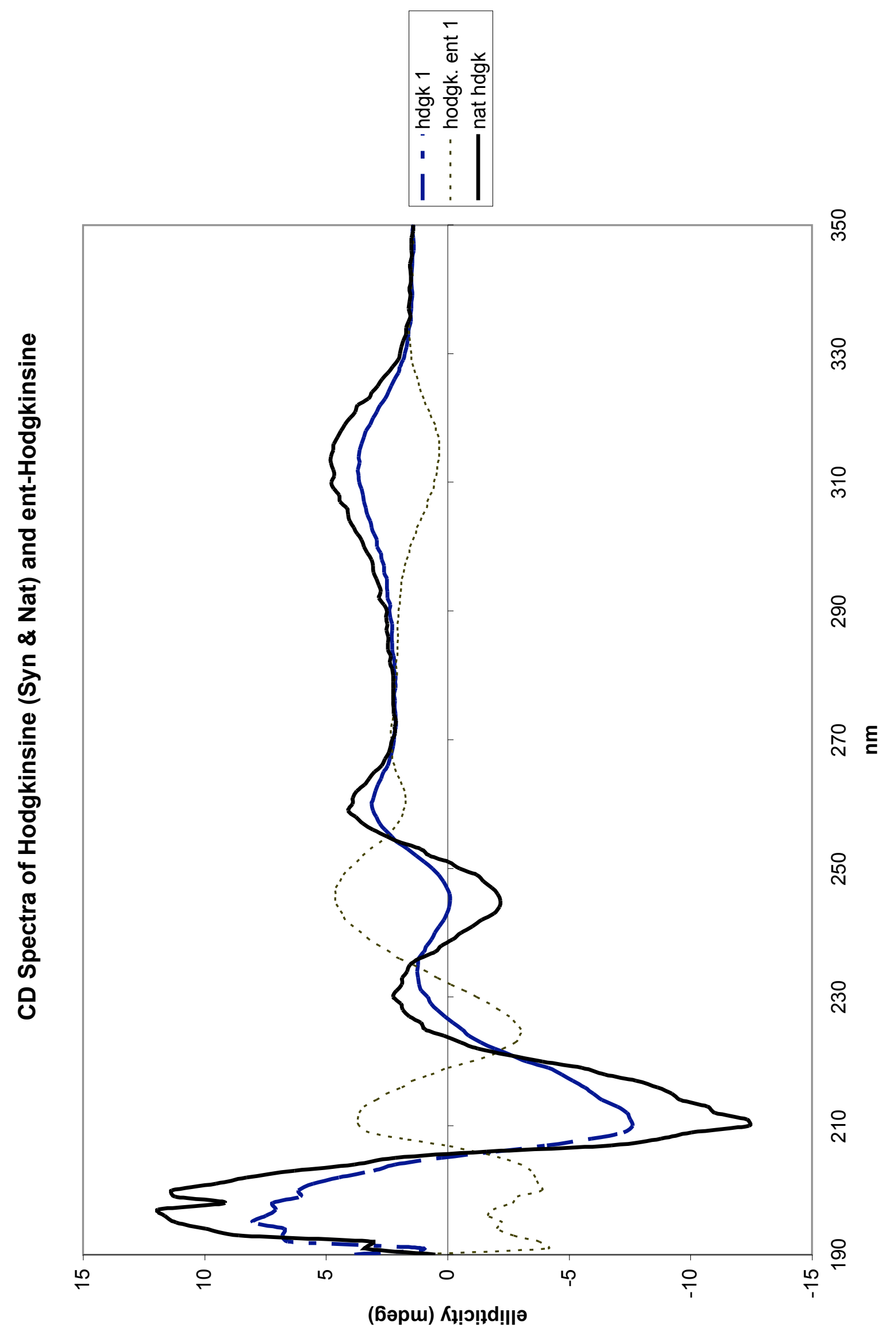




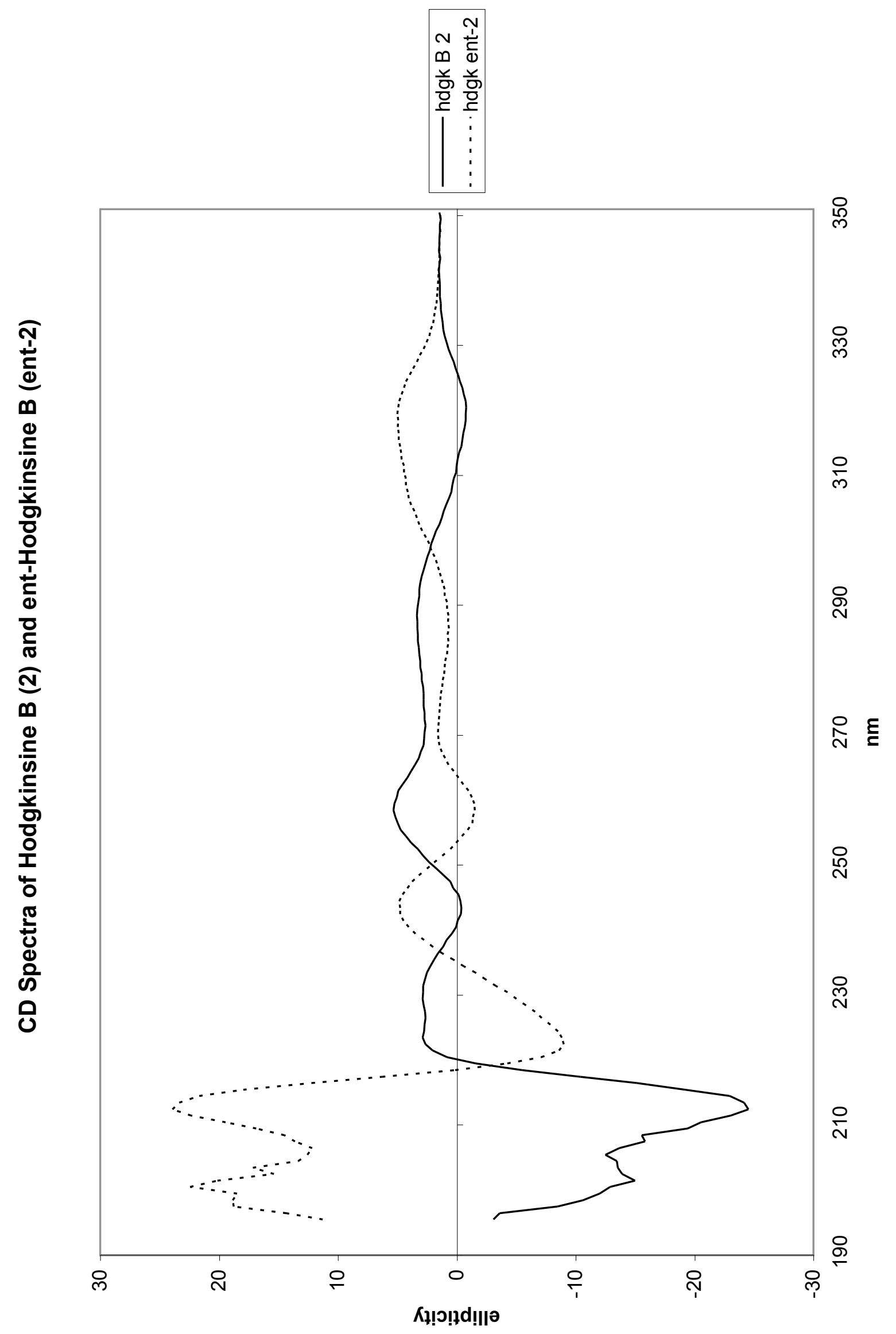




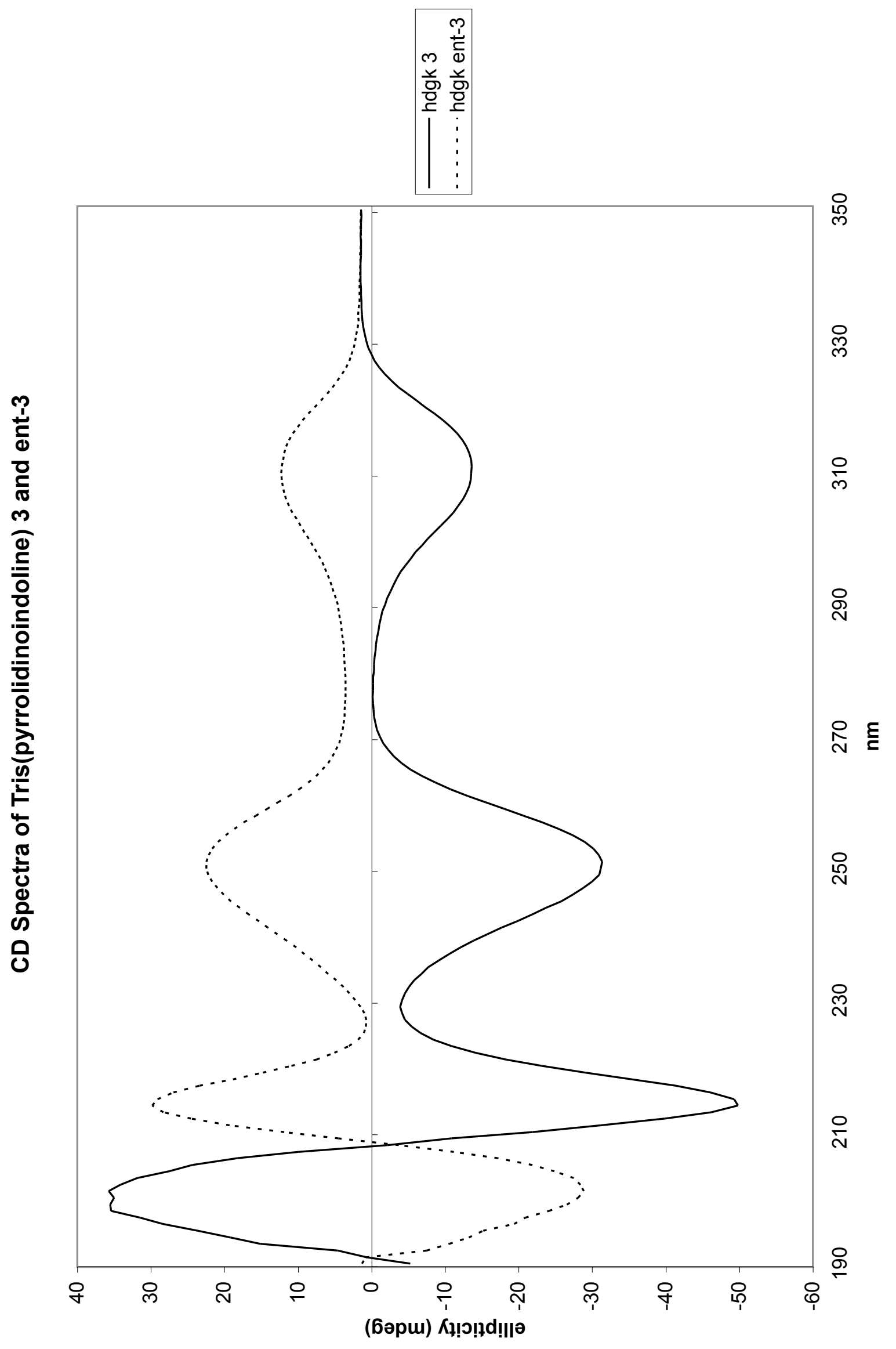




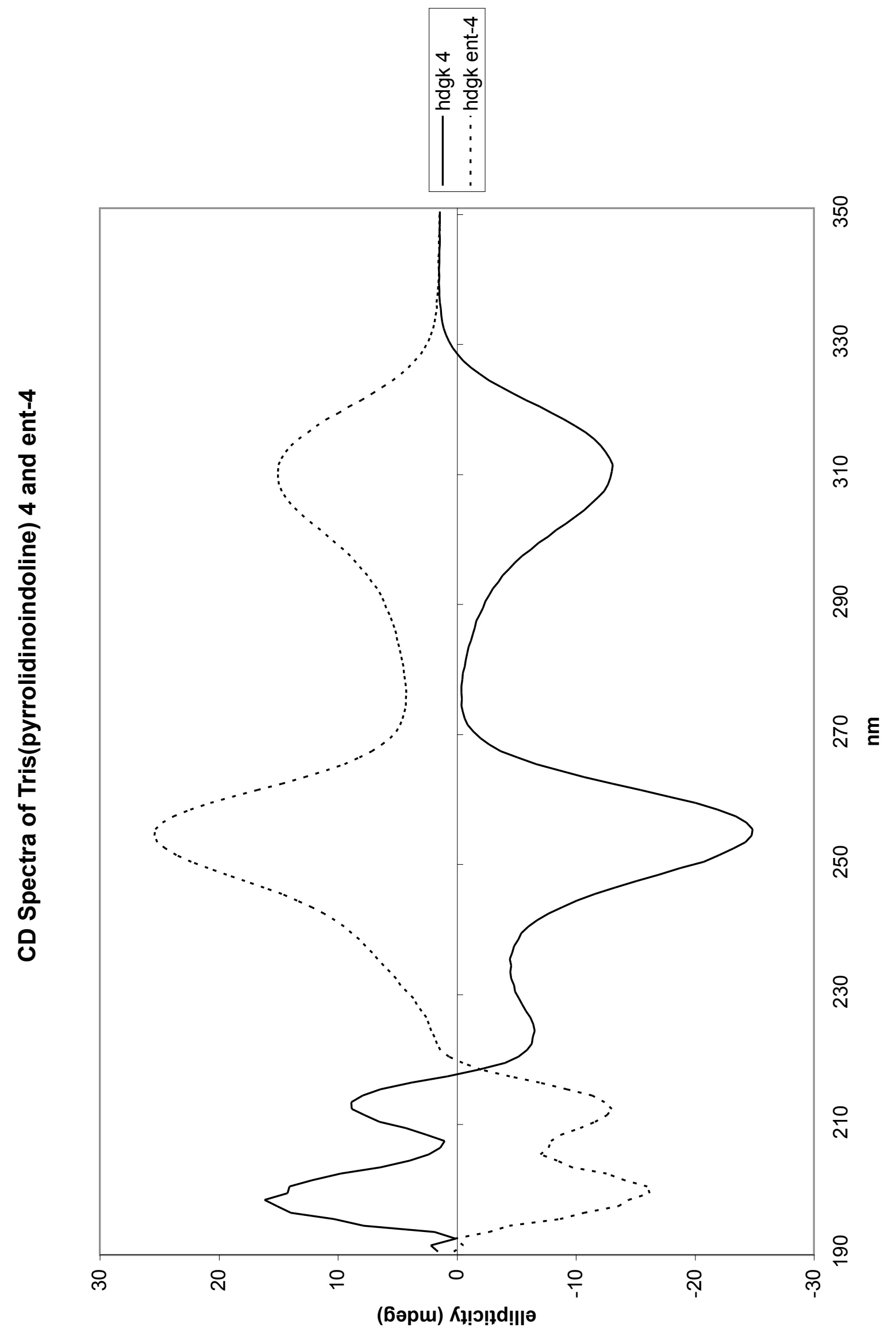

